\title{
CENTRAL FOURIER-STIELTJES TRANSFORMS WITH AN ISOLATED VALUE
}

BY

\author{
ALAN ARMSTRONG
}

\begin{abstract}
ABSTRACr. Let $\mu$ be a central Borel measure on a compact, connected group $\boldsymbol{G}$. If 0 is isolated in the range of $\hat{\mu}$, then there exists a closed, normal subgroup $H$ of $G$ such that $\pi_{H} \mu$, the restriction of $\mu$ to the cosets of $H$, is the convolution of an invertible measure with a nonzero idempotent measure. This result extends $I$. Glicksberg's result for LCA groups. An example is given which shows that this result is false in general for disconnected groups.
\end{abstract}

1. Introduction. In this paper we extend to compact groups a result obtained by I. Glicksberg [2] for LCA groups. Let $\mu$ be a bounded Borel measure on a LCA group $G$. Glicksberg proved that if 0 is an isolated value in the range of $\hat{\mu}$, then there exists a compact subgroup $H$ of $G$ such that $\pi_{H} \mu$, the restriction of $\mu$ to the cosets of $H$, is the convolution of an invertible measure with a nonzero idempotent measure. We shall prove this result for central measures on compact, connected groups, concluding additionally that $H$ is a closed normal subgroup. A counterexample to the disconnected case will also be given.

Our result generalizes D. Rider's characterization [10] of the central idempotent measures on compact groups. We will borrow rather extensively from Rider's paper and will have need to quote some results from it. Many of our lemmas and theorems are extensions of his results.

2. Preliminaries. For $G$ a compact group, $\Gamma=\Gamma(G)$ will denote the equivalence classes of irreducible unitary representations of $G$. Corresponding to each $\alpha \in \Gamma$, we denote by $\chi_{\alpha}$ the character of the class $\alpha$, by $d(\alpha)$ its degree and by $\Psi_{\alpha}$ the function $\chi_{\alpha} / d(\alpha)$. The Fourier-Stieltjes transform of a measure $\mu \in M^{z}(G)$, the center of $M(G)$, is defined by

$$
\hat{\mu}(\alpha)=\int \bar{\Psi}_{\alpha} d \mu \quad(\alpha \in \Gamma) .
$$

We shall denote by $E(\mu)$ the set $\{\alpha \in \Gamma: \hat{\mu}(\alpha) \neq 0\}$, i.e., $E(\mu)$ is the support of $\hat{\mu}$.

Let $H$ be a closed, normal subgroup of $G$. A measure $\mu$ is said to be $H$-canonical if

$$
\mu=\left(\sum_{\alpha} c_{\alpha} d(\alpha) \chi_{\alpha}\right) m_{H},
$$

where the sum is finite, $m_{H}$ denotes the Haar measure of $H$, and $1 / c_{\alpha}=\int\left|\chi_{\alpha}\right|^{2} d m_{H}$. If $\mu$ is $H$-canonical, then $\mu$ is idempotent and $E(\mu)$ consists of a finite union of

Presented to the Society, January 30, 1977; received by the editors February 21, 1979.

AMS (MOS) subject classifications (1970). Primary 43A30; Secondary 43A05.

Key words and phrases. Central measure, Fourier-Stieltjes transform, isolated value.

(C) 1980 American Mathematical Society 0002-9947/80/0000-0254/\$04.75 
hypercosets of $H$. For a discussion of canonical measures and the hypercoset structure of a compact group, see Rider [9], [10].

For $H$ a normal Borel subgroup of $G$, we define $\pi_{H} \mu \in M(G)$ by

$$
\left(\pi_{H} \mu\right)(E)=\sum_{x} \mu(E \cap x H) \quad(\mu \in M(G)),
$$

the sum being over distinct coset representatives of $H$. An argument identical to that in Rudin [11, p. 63] shows that the mapping $\mu \rightarrow \pi_{H} \mu$ is an algebra homomorphism of the convolution algebra $M(G)$. An easy computation shows that $\pi_{H}$ maps $M^{Z}(G)$ into itself.

Finally, we define $I(G)$ by $I(G)=\left\{\mu \in M^{z}(G): \mu \neq 0\right.$ and 0 is isolated in the range of $\hat{\mu}\}$. Thus, if $\mu \in I(G)$, then there exists an $\varepsilon>0$ such that if $\alpha \in \Gamma$, then $|\hat{\mu}(\alpha)| \geqslant \varepsilon$ or $\hat{\mu}(\alpha)=0$.

We can now state our main theorem.

THEOREM 1. Let $G$ be a compact, connected group and let $\mu \in I(G)$. There exists a closed, normal subgroup $H$ of $G$ such that $\pi_{H} \mu=\nu * \eta$, where $\nu$ is an invertible central measure and $\eta$ is a nonzero $H$-canonical measure.

The proof will rely on a structure theorem for compact, connected groups. A theorem of A. Weil [12, p. 91] states that every compact, connected group is a factor group of a group of the form $A \times \Pi_{I} G_{\alpha}$, where $A$ is abelian, each $G_{\alpha}$ is a compact, connected, simple Lie group and $I$ is some index set. We first prove Theorem 1 for $I$ finite, then for $I$ countable and finally for $I$ of arbitrary cardinality. We defer until later the extension to factor groups.

3. Finite products. In this section we will prove Theorem 1 for groups of the form $A \times \Pi_{1}^{n} G_{i}$, where $A$ is abelian, and each $G_{i}$ is a compact, connected, simple Lie group. We first define some properties that are satisfied by the groups $G_{i}$.

Definition. A group $G$ is said to satisfy Condition I if for all $x \notin Z(G)$, the center of $G$,

$$
\Psi_{\alpha}(x) \rightarrow 0 \text { as } d(\alpha) \rightarrow \infty
$$

A group $G$ is said to satisfy Condition II if for each positive integer $t$ there are finitely many $\beta_{i} \in \Gamma$, each $\beta_{i}$ of degree $t$, such that if $\alpha \in \Gamma$ and $d(\alpha)=t$, then $\alpha=\gamma \beta_{i}$ for some $\beta_{i}$ and $\gamma \in \Gamma$ with $d(\gamma)=1$.

These conditions were introduced by Rider in [9], where he showed that they were satisfied by the unitary groups. Using results of Ragozin [8], Rider [10] proved that all compact, connected, simple Lie groups satisfy these conditions. This implies that for these groups the number of representations of a given degree is finite since the only linear character on such groups is identically 1 . It is obvious that Condition II is also satisfied by abelian groups as well as by finite products of groups each satisfying Condition II.

If $G$ is a compact, connected simple Lie group, then $Z(G)$ is finite. The set $Z^{\perp}=\left\{\alpha \in \Gamma: \psi_{\left.\alpha\right|_{z}} \equiv 1\right\}$ is the dual object of the factor group $G / Z$. This set contains representatives of arbitrarily large degree. For if not, then $G / Z$ would 
contain an open abelian subgroup (C. C. Moore [7]), contradicting the connectedness of $G$. Using this fact one can prove that if $\gamma \in \Gamma(Z)$, then there exists a sequence $\{\alpha\} \subset \Gamma(G)$ with $d(\alpha) \rightarrow \infty$ such that $\psi_{\left.\alpha\right|_{z}}=\gamma$ for all $\alpha$ (see Rider [9]).

Now consider the collection of subsets $\left\{y x Z y^{-1}\right\}_{y \in G}$ where $x \notin Z$. We claim that this collection is infinite. If not, then the index $|G / Z: C(x Z) / Z|<\infty$ where $C(x Z)=\{y Z: x y Z=y x Z\}$, the centralizer of $x Z$ in the group $G / Z$. Since $G / Z$ is connected, we conclude that $C(x Z)=G$, i.e., $x Z \in Z(G / Z)$. Now choose $\{\alpha\} \subset Z^{\perp}$ with $d(\alpha) \rightarrow \infty$. By Condition $I,\left|\psi_{\alpha}(x)\right| \rightarrow 0$ as $d(\alpha) \rightarrow \infty$, but $\left|\psi_{\alpha}(x)\right|=$ $\left|\psi_{\alpha}(x Z)\right|=1$ since $x Z \in Z(G / Z)$, a contradiction. Now if $\mu \in M^{Z}(G)$, then $|\mu|\left(y x Z y^{-1}\right)=|\mu|(x Z)$. Since $\mu$ is bounded, it follows that $|\mu|(x Z)=0$. Hence, if $H$ is a subgroup of $Z$, then we have the equality

$$
\pi_{H} \mu=\pi_{H}\left(\mu_{\mid Z}\right)
$$

Definition. A measure $\mu \in M^{z}(G)$ is of bounded representation type (b.r.t.) if there exists a positive integer $M$ such that $\hat{\mu}(\alpha)=0$ if $d(\alpha)>M$.

The idea of the proof is to show that either $\mu_{\mid Z} \neq 0$, or else $\mu$ is of b.r.t. Throughout the remainder of this section, $A$ will denote an abelian group and $G_{i}$ a compact, connected, simple Lie group.

LEMMA 2. If $G=\Pi_{1}^{n} G_{i}$ and $\mu \in I(G)$, then there exists a closed normal subgroup $H$ of $G$ such that if $\sigma=\mu_{\mid H}$, then $\sigma \neq 0, \sigma$ is of b.r.t. and $\pi_{K} \sigma=\pi_{K} \mu$ for any subgroup $K$ of $H$.

Proof. Assume $\mu$ is not of b.r.t. Then there exists a sequence $\{\alpha\} \subset E(\mu)$ with $d(\alpha) \rightarrow \infty$, where $\alpha=\alpha_{1} \cdots \alpha_{n}, \alpha_{i} \in \Gamma\left(G_{i}\right)$. Hence, for some $i, d\left(\alpha_{i}\right) \rightarrow \infty$. Assume $i=1$. Let $H_{1}=Z\left(G_{1}\right) \times G_{2} \times \cdots \times G_{n}$ and let $\sigma_{1}=\mu_{\mid H_{1}}$. We claim that $\sigma_{1} \neq 0$ and $\sigma_{1} \in I\left(H_{1}\right)$. Now

$$
\int \bar{\Psi}_{\alpha} d \sigma_{1}=\int_{G} \bar{\Psi}_{\alpha} d \mu-\int_{G-H_{1}} \bar{\Psi}_{\alpha} d \mu
$$

Since $G_{1}$ satisfies Condition I, the Lebesgue Dominated Convergence Theorem implies that the last integral in (2) converges to 0 . Since $\alpha \in E(\mu)$ and $\mu \in I(G)$, it follows that $\sigma_{1} \neq 0$.

Now let $\gamma \in \Gamma\left(Z_{1}\right)$ and $\beta \in \Gamma\left(\Pi_{2}^{n} G_{i}\right)$. Choose a sequence $\{\lambda\} \subset \Gamma\left(G_{1}\right)$ with $d(\lambda) \rightarrow \infty$ such that $\Psi_{\lambda \mid z_{1}}=\gamma$. Then

$$
\int_{H_{1}} \bar{\lambda} \bar{\Psi}_{\beta} d \sigma_{1}=\int_{G} \bar{\Psi}_{\lambda} \bar{\Psi}_{\beta} d \mu-\int_{G-H_{1}} \bar{\Psi}_{\lambda} \bar{\Psi}_{\beta} d \mu
$$

As before, the last integral in (3) converges to 0 as $d(\lambda) \rightarrow \infty$. Since $\mu \in I(G)$, it follows that $\sigma_{1} \in I\left(H_{1}\right)$.

If $\sigma_{1}$ is not of b.r.t., then we can repeat the above process. Thus we obtain a subgroup $H=\Pi_{1}^{n} H_{i}$, where $H_{i}=Z\left(G_{i}\right)$ or $G_{i}$, and a measure $\sigma \in I(H)$ of b.r.t. If $K$ is a normal subgroup of $H$, then $K$ is a normal subgroup of $G$, and the argument that was used to prove (1) can be easily generalized to show that $\pi_{K} \mu=\pi_{K} \sigma$.

Lemma 2 allows us to restrict ourselves to measures of b.r.t. We shall complete the section by showing that such measures can be written as a finite sum of 
measures (with character coefficients) each of which can be viewed as a measure on an abelian factor group for which Glicksberg's theorem holds.

Let $H$ be a closed, normal subgroup of $G$ and let $\pi$ be the natural projection of $G$ onto $G / H$. If $\mu \in M(G)$, we define the projection $\mu^{*}$ of $\mu$ onto $G / H$ by the equation

$$
\int_{G / H} f d \mu^{*}=\int_{G} f(\pi(x)) d \mu \quad(f \in C(G / H)) .
$$

The transform of $\mu$ agrees with the transform of $\mu^{*}$ on $H^{\perp}$. Thus, if $\hat{\mu}$ vanishes off $H^{\perp}$, then $\mu$ can be identified with a measure on $G / H$. This will be the case if $\mu$ satisfies the equality $\mu=\mu * m_{H}$. Also, if $\nu$ is a measure on $G / H$, then there is a measure $\mu$ on $G$ such that $\mu^{*}=\nu$ and $\mu=\mu * m_{H}$.

LEMMA 3. Let $H$ be a closed normal subgroup of $G$ and let $K$ be a subgroup of $G$ such that $H \subseteq K \subseteq G$. If $\mu \in M(G)$, then $\left(\pi_{K} \mu\right)^{*}=\pi_{K / H} \mu^{*}$.

Proof.We shall first show that if $\mu$ is supported on $x K$, then $\mu^{*}$ is supported on $\pi(x K)$. Let $U^{\prime}$ be an open set in $G / H$ such that $\bar{U}^{\prime} \cap \pi(x K)=\varnothing$, and let $f^{\prime}$ be a continuous function supported on $U^{\prime}$. If we define $U=\pi^{-1}\left(U^{\prime}\right)$ and $f(x)=$ $f^{\prime}(\pi(x))$, then $U$ is open in $G, \bar{U} \cap x K=\varnothing$, and $f$ is a continuous function supported on $U$. Thus $\int_{G / H} f^{\prime} d \mu^{*}=0$, which implies that $\left|\mu^{*}\right|\left(U^{\prime}\right)=0$ and hence that $\mu^{*}$ is supported on $\pi(x K)$.

Next, we show that if $\mu$ vanishes on Borel subsets of the coset $x K$, then $\mu^{*}$ vanishes on Borel subsets of the coset $\pi(x K)$. Let $\varepsilon>0$. By the regularity of $\mu$ there is an open set $U \supset x K$ such that $|\mu|(U)<\varepsilon$. Let $U^{\prime}=\pi(U)$ and let $f^{\prime}$ be a continuous function supported on $U^{\prime}$ such that $\left\|f^{\prime}\right\|_{\infty}<1$. If $f(x)=f^{\prime}(\pi(x))$, then

$$
\left|\int_{G / H} f^{\prime} d \mu^{*}\right|=\left|\int_{G} f d \mu\right|<\varepsilon .
$$

Thus, $\left|\mu^{*}\right|\left(U^{\prime}\right)<\varepsilon$, and hence $\left|\mu^{*}\right|(\pi(x K))=0$ by regularity.

Now if $\mu \in M(G)$, then $\mu=\pi_{K} \mu+\sigma$, where $\sigma$ vanishes on Borel subsets of cosets of $K$, and $\mu^{*}=\pi_{K / H} \mu^{*}+\sigma_{0}$, where $\sigma_{0}$ vanishes on the Borel subsets of cosets of $K / H$. Thus $\pi_{K / H} \mu^{*}+\sigma_{0}=\mu^{*}=\left(\pi_{K} \mu\right)^{*}+\sigma^{*}$. But $\left(\pi_{K} \mu\right)^{*}$ is supported on cosets of $K / H$ and $\sigma^{*}$ vanishes on cosets of $K / H$, and thus $\pi_{K / H} \mu^{*}=\left(\pi_{K} \mu\right)^{*}$ by the uniqueness of the decomposition.

LEMMA 4. If $G=A \times \Pi_{1}^{n} G_{i}$ and $\mu \in I(G)$ is of b.r.t., then $\mu=\Sigma_{1}^{n} d\left(\beta_{i}\right) \chi_{\beta_{i}} \lambda_{i}$, where each $\lambda_{i}$ satisfies $\lambda_{i}=\lambda_{i} * m_{G^{\prime}}$ with $G^{\prime}=\Pi_{1}^{n} G_{i}$.

Proof. Let $\beta_{i} \in \Gamma\left(G^{\prime}\right)$ and put $\theta_{i}=d\left(\beta_{i}\right) \chi_{\beta_{i}} m_{G^{\prime}}$. Then $\theta_{i}$ is a central idempotent and $E\left(\theta_{i}\right)=\beta_{i} \Gamma(A)$. Since $\mu$ is of b.r.t. and $G$ satisfies Condition II, $\mu$ is a finite sum of measures of the form $\mu * \theta_{i}$. Put $\lambda_{i}=\psi_{\beta_{i}} \mu * m_{G^{\prime}}$. An elementary calculation (see Rider [9]) shows that $\mu * \theta_{i}=d\left(\beta_{i}\right) \chi_{\beta_{i}} \lambda_{i}$.

It is clear in the proof of Lemma 4 that 0 is an isolated value in the range of each $\hat{\lambda}_{i}$. Since $\lambda_{i}=\lambda_{i} * m_{G^{\prime}}$, we can view $\lambda_{i}$ as an element of $I(A)$ and apply Glicksberg's result to $\lambda_{i}$. However, the $\lambda_{i}$ may yield different $H_{i}$. The next lemma deals with this problem. 
Lemma 5. Let $\lambda_{i} \in I(A)$ for $1 \leqslant i \leqslant n$, then there exists a compact subgroup $H$ of $A$ such that, for all $i, \pi_{H} \lambda_{i}=\nu_{i} * \eta_{i}$, where $\nu_{i}$ is invertible and $\eta_{i}$ is $H$-canonical, and at least one $\eta_{i} \neq 0$.

Proof. We will assume $n=2$, the general case following easily by induction. We apply Glicksberg's result to $\lambda_{1}$ and $\lambda_{2}$, so that there exist compact subgroups $H$ and $K$ such that $\pi_{H} \lambda_{1}=\nu_{1} * \eta_{1}$ and $\pi_{K} \lambda_{2}=\nu_{2} * \eta_{2}$, where $\eta_{1}$ is $H$-canonical and $\eta_{2}$ is $K$-canonical. The proof is now divided into three cases.

Case I. $H \cap K$ is open in $H$ and in $K$.

In this case, $\pi_{H}=\pi_{H K}=\pi_{K}$, and it is easily seen that we can replace both $H$ and $K$ by $H K$.

Case II. $H \cap K$ is open in $H$ but not in $K$.

In this case, $\pi_{H}=\pi_{H \cap K}=\pi_{H K}$. The last equality is true for any two subgroups of $A$. Thus

$$
\pi_{H} \lambda_{2}=\pi_{H} \pi_{K} \lambda_{2}=\pi_{H}\left(\nu_{2} * \eta_{2}\right)=\pi_{H} \nu_{2} * \pi_{H} \eta_{2}=0
$$

since $\pi_{H} m_{K}=0$.

The case where $H \cap K$ is open in $K$ but not in $H$ is handled identically.

Case III. $H \cap K$ is open neither in $H$ nor in $K$.

Clearly, we can assume $\pi_{K} \lambda_{1} \neq 0$. A theorem of Glicksberg and Wik [3] states that the range of $\left(\pi_{K} \lambda_{1}\right)$ is contained in the closure of the range of $\hat{\lambda}_{1}$. Thus $\pi_{K} \lambda_{1} \in I(A)$, so that there exists a subgroup $L$ of $A$ such that $0 \neq \pi_{L} \pi_{K} \lambda_{1}=\nu * \eta$, where $\eta$ is $L$-canonical. Since $\pi_{K} \eta \neq 0$, it follows that $L \cap K$ is open in $L$. Thus $\pi_{L} \lambda_{1}=\pi_{L} \pi_{K} \lambda_{1}=\nu * \eta$. If $\pi_{L} \lambda_{2} \neq 0$, then $0 \neq \pi_{L} \lambda_{2}=\pi_{L} \pi_{K} \lambda_{2}=\pi_{L}\left(\nu_{2} * \eta_{2}\right)$. Hence $\pi_{L} \eta_{2} \neq 0$ and $L \cap K$ is open in $K$. The problem has now been reduced to Case I.

THEOREM 6. If $G=A \times \Pi_{1}^{n} G_{i}$ and $\mu \in I(G)$ is of b.r.t., then there exists a closed, normal subgroup $H$ of $G$ such that $0 \neq \pi_{H} \mu=\nu * \eta$, where $\nu$ is invertible and $\eta$ is H-canonical.

Proof. By Lemma $4, \mu=\Sigma_{1}^{m} d\left(\beta_{i}\right) x_{\beta_{i}} \lambda_{i}$, where $\lambda_{i}=\psi_{\beta_{i}} \mu * m_{G^{\prime}}$ for all $i$. The projection $\lambda_{i}^{*}$ of $\lambda_{i}$ onto $G / G^{\prime}$ is an element of $I\left(G / G^{\prime}\right)$, and so by Lemma 5 (since $G / G^{\prime} \simeq A$ ) there exists a closed, normal subgroup $H / G^{\prime}$ of $G / G^{\prime}$ such that $\pi_{H / G^{\prime}} \lambda_{i}^{*}=\nu_{i} * \eta_{i}$, where $\eta_{i} \ll m_{H / G^{\prime}}$ and $\nu_{i} \in M^{-1}\left(G / G^{\prime}\right)$ for all $i$. Since at least one $\eta_{i} \neq 0$, we can assume $\eta_{i} \neq 0$ for all $i$. Identify $\nu_{i}$ and $\eta_{i}$ with measures on $G$, so that $\nu_{i} * m_{G^{\prime}}=\nu_{i}$ and $\eta_{i} * m_{G^{\prime}}=\eta_{i}$. Thus $\eta_{i} \ll m_{H}$ for all $i$ and there exist measures $\nu_{i}^{\prime}$ on $G$ such that $\nu_{i} * \nu_{i}^{\prime}=m_{G^{\prime}}$. (Note that $m_{G^{\prime}}$ is identified with the identity measure in $M\left(G / G^{\prime}\right)$.)

Now by Lemma $3,\left(\pi_{H} \lambda_{i}\right)^{*}=\pi_{H / G^{\prime}} \lambda_{i}^{*}$. Thus, $\pi_{H} \lambda_{i} * m_{G^{\prime}}=\nu_{i} * \eta_{i}$. This implies that $\pi_{H} \lambda_{i} \neq 0$, and since $\lambda_{i} * m_{G^{\prime}}=\lambda_{i}$, we have

$$
0 \neq \pi_{H} \lambda_{i}=\pi_{H} \lambda_{i} * \pi_{H} m_{G^{\prime}}=\pi_{H} \lambda_{i} * m_{G^{\prime}}=\nu_{i} * \eta_{i} \text {. }
$$

Therefore,

$$
\pi_{H} \mu=\sum_{1}^{m} d\left(\beta_{i}\right) \chi_{\beta_{i}}\left(\nu_{i} * \eta_{i}\right)
$$


Put $\nu=\Sigma_{1}^{m} d\left(\beta_{i}\right) \chi_{\beta_{i}} \nu_{i}$ and $\eta=\sum_{1}^{m} d\left(\beta_{i}\right) \chi_{\beta_{i}} \eta_{i}$. If $\gamma \in \Gamma(A)$, then

$$
\begin{aligned}
\left(\pi_{H} \mu\right)^{\wedge}\left(\gamma \beta_{i}\right) & =\left(d\left(\beta_{i}\right) \chi_{\beta_{i}}\left(\nu_{i} * \eta_{i}\right)\right)^{\wedge}\left(\gamma \beta_{i}\right) \\
& =\int \bar{\gamma}\left|\chi_{\beta_{i}}\right|^{2} d\left(\nu_{i} * \eta_{i}\right) \\
& =\int \bar{\gamma} d\left(\nu_{i} * \eta_{i}\right)=\hat{\nu}_{i}(\gamma) \hat{\eta}_{i}(\gamma) .
\end{aligned}
$$

On the other hand,

$$
\begin{aligned}
(\nu * \eta)^{\wedge}\left(\gamma \beta_{i}\right) & =\left(d\left(\beta_{i}\right) \chi_{\beta_{i}} \nu_{i}\right)^{\wedge}\left(\gamma \beta_{i}^{*}\right) \cdot\left(d\left(\beta_{i}\right) \chi_{\beta_{i}} \eta_{i}\right)^{\wedge}\left(\gamma \beta_{i}\right) \\
& =\int \bar{\gamma}\left|\chi_{\beta_{i}}\right|^{2} d \nu_{i} \cdot \int \bar{\gamma}\left|\chi_{\beta_{i}}\right|^{2} d \eta_{i}=\hat{\nu}_{i}(\gamma) \hat{\eta}_{i}(\gamma)
\end{aligned}
$$

Therefore, $\pi_{H} \mu=\nu * \eta$, and if we now let $\eta_{i}^{\prime}=d\left(\beta_{i}\right) \chi_{\beta_{i}} \eta_{i}$ and $\nu_{0}=\nu+$ $\Pi_{1}^{m}\left(\delta_{0}-\eta_{i}^{\prime}\right)$, then $\pi_{H} \mu=\nu_{0} * \eta$. It is clear that $\eta \ll m_{H}$ and the inverse of $\nu_{0}$ is given by $\nu_{0}^{-1}=\Sigma_{1}^{m} d\left(\beta_{i}\right) x_{\beta_{i}} \nu_{i}^{\prime}+\Pi_{1}^{m}\left(\delta_{0}-\eta_{i}^{\prime}\right)$.

COROLlaRY 7. If $G=\Pi_{1}^{n} G_{i}$ and $\mu \in I(G)$ is of b.r.t. then $\mu$ is a trigonometric polynomial.

4. Countable products. In this section we consider the case when $G=A \times \Pi_{1}^{\infty} G_{i}$, where $A$ and $G_{i}$ are as in the preceding section. If $\mu \in M^{2}(G)$, then we shall say that $\mu$ is of bounded representation type in each coordinate if there exist positive integers $M_{i}, 1 \leqslant i \leqslant \infty$, such that if $\alpha=\alpha_{1} \cdots \alpha_{n} \in E(\mu)$ and $d\left(\alpha_{i}\right)>M_{i}$ for some $i$, then $\hat{\mu}(\alpha)=0$. We shall first reduce the problem to measures which are of b.r.t. in each coodinate. We then shall prove that if $\mu$ is such a measure and $\mu \in I(G)$, then there exists an $N$ such that $\mu=\mu * m_{H^{\prime}}$ where $H=\Pi_{N+1}^{\infty} G_{i}$. Hence we can treat $\mu$ as a measure on $A \times \Pi_{1}^{N} G_{i}$ and apply the results of the previous section.

We note that by replacing $\mu$ by $\mu * \tilde{\mu}$, where $\tilde{\mu}(E)=\overline{\mu\left(E^{-1}\right)}$, we can assume that $\hat{\mu}$ is positive. It is also clear that we can assume $\hat{\mu}(\alpha)>1$ for all $\alpha \in E(\mu)$. If $\mu$ is not of b.r.t. in the $i$ th coodinate, put $H_{i}=Z_{i}$; otherwise put $H_{i}=G_{i}$. Let $K_{n}=A$ $\times H_{1} \times \cdots \times H_{n} \times G_{n+1} \times \cdots$ and $\sigma_{n}=\mu_{\mid K_{n}}$. The argument given in Lemma 2 shows that $\sigma_{n} \neq 0$ and that $\hat{\sigma}_{n}(\alpha) \geqslant 1$ for all $\alpha \in E\left(\sigma_{n}\right)$. Thus $\left\|\sigma_{n}\right\|>1$, i.e., $|\mu|\left(K_{n}\right) \geqslant 1$. Put $K=\bigcap_{n=1}^{\infty} K_{n}=A \times \Pi_{1}^{\infty} H_{i}$ and put $\sigma=\mu_{\mid K}$. Since $|\mu|(K)=$ $\lim _{n \rightarrow \infty}|\mu|\left(K_{n}\right) \geqslant 1$, it follows that $\sigma \neq 0$.

We claim that $\sigma \in I(K)$. Let $\alpha=\gamma \alpha_{1} \cdots \alpha_{n} \in \Gamma(K)$. If $H_{i}=Z_{i}$, choose $\left\{\lambda_{i}^{j}\right\}_{j=1}^{\infty} \subset \Gamma\left(G_{i}\right)$ such that $d\left(\lambda_{i}^{j}\right) \rightarrow \infty$ as $j \rightarrow \infty$ and such that $\Psi_{\lambda / \mid z_{i}}=\alpha_{i}$ if $i<n$ and $\Psi_{\lambda \gamma^{\prime} \mid Z_{i}}=1$ if $i>n$. If $H_{i}=G_{i}$, choose $\left\{\lambda_{i}^{j}\right\} \subset \Gamma(G)$ such that $\lambda_{i}^{j}=\alpha_{i}$ for all $j$. Now put $\beta_{m}=\lambda_{1}^{m} \cdots \lambda_{m}^{m}$ for $m \geqslant n$. Now if $x \notin K$ then $x_{i} \notin Z_{i}$ for some $i$ for which $H_{i}=Z_{i}$, so that $\lim _{m \rightarrow \infty} \Psi_{\beta_{m}}(x)=0$. Since $\Psi_{\beta_{m} \mid K}=\Psi_{\alpha}$, we have the equality

$$
\int \bar{\Psi}_{\alpha} d \sigma=\int \bar{\Psi}_{\beta_{m}} d \sigma=\int \bar{\Psi}_{\beta_{m}} d \mu-\int \bar{\Psi}_{\beta_{m}} d \mu_{\mid G-K}
$$


The last integral above converges to 0 by the Lebesgue Dominated Convergence Theorem. Since $\mu \in I(G)$, it follows that $\sigma \in I(K)$, and since $\pi_{K} \mu=\pi_{K} \sigma$, we may assume $\mu$ is of b.r.t. in each coodinate.

Our next lemma deals with norms of polynomials and generalizes a result of Rider [10, p. 466]. Our proof is a slight modification of his.

LEMMA 8. Let $G$ be a compact, connected group and let $P=\Sigma c_{\alpha} d(\alpha) \chi_{\alpha}$ be a central polynomial on $G$ with $c_{\alpha} \geqslant 1$ for all $\alpha$. If $\|P\|_{1}<1+1 / 300$, then $P=c \gamma$, where $\gamma$ is a linear character and $1 \leqslant c \leqslant 1+1 / 300$.

Proof. Write $|P|^{2}=P \bar{P}=\sum a_{\alpha} \chi_{\alpha}, a_{\alpha}>1$, and $|P|^{4}=b_{\alpha} \chi_{\alpha}, b_{\alpha}>1$. Let

$$
\begin{aligned}
M & =\|P\|_{2}^{2}=\int P \bar{P}=\sum \int c_{\alpha}^{2} d^{2}(\alpha) x_{\alpha} \bar{x}_{\alpha} \\
& =\sum c_{\alpha}^{2} d^{2}(\alpha)>\sum c_{\alpha} d^{2}(\alpha)=\|P\|_{\infty} .
\end{aligned}
$$

We have the inequalities

$$
a_{\alpha}=\int|P|^{2} \bar{\chi}_{\alpha} \leqslant d(\alpha)\|P\|_{2}^{2}=M d(\alpha)
$$

and

$$
b_{\alpha}=\int|P|^{4} \bar{\chi}_{\alpha} \leqslant d(\alpha)\|P\|_{\infty}^{2} \int|P|^{2} \leqslant d(\alpha) M^{2} \cdot M=d(\alpha) M^{3} .
$$

By Hölder's inequality,

$$
\sum a_{\alpha} b_{\alpha}=\int|P|^{6} \geqslant M^{5}\|P\|_{1}^{-4}
$$

Define

$$
A_{1}(g)=M^{-4} \sum b_{\alpha}\left(1-a_{\alpha}(M d(\alpha))^{-1}\right) \chi_{\alpha}(g)
$$

and

$$
A_{2}(g)=M^{-2} \sum a_{\alpha}\left(1-b_{\alpha}\left(M^{3} d(\alpha)\right)^{-1}\right) \chi_{\alpha}(g)
$$

Then

$$
\left\|A_{i}\right\|_{\infty}=A_{i}(e) \leqslant 1-\|P\|_{1}^{-4} \quad(i=1,2) .
$$

Thus if $\|P\|_{1} \leqslant 1+1 / 300$, then $\left\|A_{i}\right\|_{\infty} \leqslant 1 / 60$. It follows that $1 / 30>A_{2}-A_{1}=$ $|P|^{2} / M^{2}-|P|^{4} / M^{4}>0$. Thus either $|P(g)|<M / 5$ or $|P(g)|>4 M / 5$. Suppose $P(e)=\Sigma c_{\alpha} d^{2}(\alpha) \leqslant M / 5$. Then

$$
0 \leqslant \frac{\sum c_{\alpha}^{2} d^{2}(\alpha)}{5}-\sum c_{\alpha} d^{2}(\alpha)=\sum c_{\alpha} d^{2}(\alpha)\left(\frac{c_{\alpha}}{5}-1\right) .
$$

Since $c_{\alpha}<\|P\|_{1}<5, \Sigma c_{\alpha} d^{2}(\alpha)\left(c_{\alpha} / 5-1\right)<0$, a contradiction. Since $G$ is connected, $P(g) \geqslant 4 M / 5$ for all $g \in G$. Thus $1+1 / 300>\|P\|_{1}>4 M / 5$, which yields $M<2$, and the result follows.

LeMMA 9. Let $G=\Pi_{1}^{\infty} G_{i}$ and let $\mu \in I(G)$ be of b.r.t. in each coordinate. If $\alpha \in E(\mu)$ if and only if $\left\|\left(\Psi_{\alpha}-1\right) \mu\right\|<1 / 600$, then $\mu=c m_{G}$ for some constant $c$. 
Proof. Since $|\hat{\mu}(\alpha)-\hat{\mu}(1)|<1 / 600$ for all $\alpha \in E(\mu)$, we can assume $1<\mu(\alpha)$ $<1+1 / 600$ for all $\alpha \in E(\mu)$. Let $G^{r}=\Pi_{1}^{r} G_{i}$ and $G^{r}=\Pi_{r+1}^{\infty} G_{i}$, and let $\mu_{r}=$ $\mu * m_{G^{\prime \prime}}$. We shall show that $\mu_{r}^{*}$, which we can identify as an element of $I\left(G^{r}\right)$, is of the form $c_{r} m_{G^{r}}$ for some constant $c_{r}$. It follows that $\mu_{r}=c_{r} m_{G^{r}} * m_{G^{r}}$. The result follows since $\mu_{r} \rightarrow \mu, w^{*}$, and $c_{r} m_{G^{r}} * m_{G^{\prime \prime}} \rightarrow c m_{G}, w^{*}$, for some constant $c$.

Since $\mu$ is of b.r.t. in each coodinate, it follows that $\mu_{r}^{*}$ is of b.r.t. By Corollary 7, $\mu_{r}^{*}$ is a polynomial $P=\Sigma c_{\alpha} d(\alpha) \chi_{\alpha}$ on $G_{r}$. If $\alpha \in E\left(\mu_{r}^{*}\right)$, then $\left\|\left(\bar{\Psi}_{\alpha}-1\right) \mu_{r}^{*}\right\|<$ $1 / 600$. Now

$$
\begin{aligned}
\int\left|\left(\frac{P}{\|P\|_{\infty}}-1\right) P\right| d m_{G_{r}} & =\int \frac{|(P-P(e)) P|}{\|P\|_{\infty}} d m_{G_{r}} \\
& =\frac{1}{\|P\|_{\infty}} \int\left|\left(\sum c_{\alpha} d(\alpha) x_{\alpha}-\sum c_{\alpha} d^{2}(\alpha)\right) P\right| d m_{G_{r}} \\
& =\frac{1}{\|P\|_{\infty}} \int\left|\left(\sum c_{\alpha} d^{2}(\alpha)\left(\frac{\chi_{\alpha}}{d(\alpha)}-1\right)\right) P\right| d m_{G_{r}} \\
& \leqslant 1 / 600 .
\end{aligned}
$$

On the other hand

$$
\int\left|\left(\frac{P}{P(e)}-1\right) P\right| \geqslant \int|P|-\frac{\int|P|^{2}}{P(e)}=\|P\|_{1}-\frac{\sum c_{\alpha}^{2} d^{2}(\alpha)}{\sum c_{\alpha} d^{2}(\alpha)} .
$$

Therefore,

$$
\begin{aligned}
\|P\|_{1} & \leqslant \frac{1}{600}+\frac{\sum c_{\alpha}^{2} d^{2}(\alpha)}{\sum c_{\alpha} d^{2}(\alpha)} \\
& \leqslant \frac{1}{600}+\frac{\sum(1+1 / 600) c_{\alpha} d^{2}(\alpha)}{\sum c_{\alpha} d^{2}(\alpha)}=1+\frac{1}{300} .
\end{aligned}
$$

By Lemma $8, P=c_{r} m_{G_{r}}$ for some constant $c_{r}$.

Our next lemma is of a very technical nature. It generalizes Rider's Lemma 6.3 of [10]. First, we need to introduce the concept of an irreducible sequence.

Definition. A sequence $\left\{\alpha_{i}\right\} \subset \Gamma(G)$ is said to be irreducible if, for fixed $\beta \in \Gamma$, $\alpha_{i} \otimes \beta$ is eventually irreducible. Let $G=\Pi_{1}^{\infty} G_{i}$ and let $\left\{\alpha_{i}\right\} \subset \Gamma(G)$, with $\alpha_{i}=$ $\alpha_{i}^{(1)} \alpha_{i}^{(2)} \ldots \alpha_{i}^{(n)}$ where $\alpha_{i}^{(j)} \in \Gamma\left(G_{j}\right)$. The sequence $\left\{\alpha_{i}\right\}$ is called an $F$-sequence if for each positive integer $N$ there exists a positive integer $N^{\prime}$ such that if $i>N^{\prime}$ then $d\left(\alpha_{i}^{(j)}\right)=1$ for all $j<N$. An $F$-sequence is an irreducible sequence.

LeMma 10. Let $G=A \times \Pi_{1}^{\infty} G_{i}$, let $n$ be a positive integer, and let $M<\infty$. There exists $a \delta>0$ such that if $\mu \in I(G)$ satisfies the following:

(a) $\|\mu\|<M$.

(b) There exists a set $B$ of Borel homomorphisms $f$ into the unit circle. Each $f \in B$ is defined on a normal Borel subgroup $T_{f}$ of $G$ with $|\mu|\left(T_{f}^{c}\right)=0$. 
(c) The function $f_{1}=1 \in B$, and if $f \in B$ then there is an $F$-sequence $\{\alpha\} \subset E(\mu)$ with $\bar{\Psi}_{\alpha} \mu \rightarrow f \mu$ in norm, and for every $F$-sequence $\{\alpha\} \subset E(u), \bar{\Psi}_{\alpha} \mu \rightarrow f \mu$ in norm (for a subsequence of $\{\alpha\}$ ) for some $f \in B$.

(d) The set $B$ is partitioned into finitely many subsets $B_{1}, \ldots, B_{n}$ by $f \sim g$ if $E(f \mu)=E(g \mu)$.

(e) $\alpha \in E(\mu)$ if and only if $\left\|\left(\bar{\Psi}_{\alpha}-f\right) \mu\right\|<\delta$ for some $f \in B$, then there exist $h_{i} \in L^{\infty}(|\mu|), 1 \leqslant i \leqslant n$, such that if $\sigma=h_{1} \mu * \cdots * h_{n} \mu$, then $\left\|\left(\bar{\Psi}_{\alpha}-1\right) \sigma\right\|<1 / 600$ if and only if $\alpha \in E(\sigma)$.

Proof. We shall first show that if $f, g$ and $h \in B$ with $f \sim g$, then $E(f h \mu)=$ $E(g h \mu)$. Note that we do not claim that $f h \in B$. Since $\|f h \mu\|=\|\mu\|$, we have that $f h \mu \neq 0$. Let $\bar{\Psi}_{\beta} \mu \rightarrow g \mu$ and $\bar{\Psi}_{\gamma} \mu \rightarrow h \mu$. If $\alpha \in E(f h \mu)$, then $0 \neq \int \bar{\Psi}_{\alpha}$ fh $d \mu=$ $\lim _{\gamma} \int \bar{\Psi}_{\alpha} \bar{\Psi}_{\gamma} f d \mu$. Thus, for $\gamma>\gamma_{0}, \int \bar{\Psi}_{\alpha} \bar{\Psi}_{\gamma} f d \mu \neq 0$ and $\alpha \otimes \gamma$ is irreducible. This implies that $\alpha \gamma \in E(f \mu)=E(g \mu)$, so that $0 \neq \int \bar{\Psi}_{\alpha} \bar{\Psi}_{\gamma} g d \mu=\lim _{\beta} \int \bar{\Psi}_{\alpha} \bar{\Psi}_{\gamma} \bar{\Psi}_{\beta} d \mu$ if $\gamma>\gamma_{0}$. Since $\alpha \otimes \gamma \otimes \beta$ is irreducible for $\beta$ large enough, $\int \bar{\Psi}_{\alpha} \bar{\Psi}_{\gamma} g d \mu>1$. Thus, $\int \bar{\Psi}_{\alpha} h g d \mu=\lim _{\gamma} \int \Psi_{\alpha} \Psi_{\gamma} g d \mu>1$, i.e., $\alpha \in E(g h \mu)$. Similarly, $E(g h \mu) \subset E(f h \mu)$.

Let $\Lambda=B_{1}$ be the equivalence class containing $f_{1}=1$. If $f, g \in \Lambda$ then $E(f g \mu)=$ $E\left(f f_{1} \mu\right)=E(f \mu)=E(\mu)$, i.e., $\Lambda$ is a semigroup under function multiplication. Topologize $\Lambda$ by defining $\|f-g\|_{\Lambda}=\|f \mu-g \mu\|_{M(G)}$. If $\left\{f_{i}\right\} \subset \Lambda$, then there exists a $\nu \in M(G)$ such that $f_{i} \mu \rightarrow \nu, w^{*}$ (for some subsequence). By using a diagonal process, one can construct an $F$-sequence $\{\alpha\} \subset E(\mu)$ such that $\bar{\Psi}_{\alpha} \mu \rightarrow \nu$, $w^{*}$. From (c), $\bar{\Psi}_{\alpha} \mu \rightarrow f \mu$ in norm (for a subsequence) for some $f \in B$. Thus $\nu=f \mu$, and from (b), $\|\nu\|=\|\mu\|$. Since $\left\{f_{i}\right\} \subset \Lambda$, it follows that $f \in \Lambda$. Using a theorem of Amemiya and Ito (see Rider [10, Lemma 7.5]), $f_{i} \mu \rightarrow \nu$ in norm, and hence $\Lambda$ is a compact abelian semigroup. It follows [5, p. 99] that $\Lambda$ is a compact group. Hence, there exists a Haar measure $m$ on $\Lambda$.

Choose fixed representatives $f_{i} \in B_{i}$ with $f_{1} \equiv 1$, and define $h_{i}$ by the vector-valued integral

$$
h_{i}=\int_{\Lambda} f_{i} f d m(f), \quad f \in \Lambda
$$

Note that $h_{i}=f_{i} \int_{\Lambda} f d m(f)=f_{i} h_{1}$. Let $\sigma=h_{1} \mu * \cdots * h_{n} \mu$. Then $\sigma \neq 0$ since $l \in \cap E\left(h_{i} \mu\right)=E(\sigma)$. Also, $\sigma \in I(G)$ since each $h_{i} \mu \in I(G)$.

Let $\beta$ be a fixed element of $E(\sigma)$. Then $\beta \in E\left(f_{i} \mu\right)$ for all $i$. If $\bar{\Psi}_{\alpha} \rightarrow f_{i}$, as in (c), then $\beta \otimes \alpha$ is eventually irreducible and $\beta \alpha \in E(\mu)$. By (e), $\left\|\left(\bar{\Psi}_{\beta} \bar{\Psi}_{\alpha}-g_{j}\right) \mu\right\|<\delta$ for some $g_{j} \in B_{j}$. Since $\bar{\Psi}_{\alpha} \rightarrow f_{i}$ a.e. $(|\mu|)$, we have that

$$
\left\|\left(\bar{\Psi}_{\beta}-\bar{f}_{i} g_{j}\right) \mu\right\|=\left\|\left(\bar{\Psi}_{\beta} f_{i}-g_{j}\right) \mu\right\|<\delta .
$$

Since $f_{1} \equiv 1$, there is a $g_{K}$ such that

$$
\left\|\left(\bar{\Psi}_{\beta}-g_{K}\right) \mu\right\|<\delta .
$$

We will show that $g_{K} \sigma=\sigma$.

From (4) and (5) it follows that (choosing $\delta<1 / 2$ )

$$
\left\|\left(g_{K}-\bar{f}_{i} g_{j}\right) \mu\right\|=\left\|\left(g_{K} f_{i}-g_{j}\right) \mu\right\|<1 .
$$


Thus $1 \in E\left(g_{K} f_{i} \mu\right)$ and hence $g_{K} f_{i} \in B_{j}$. Since $g_{K} f_{i} \sim f_{j}$, it follows that $E\left(\bar{f}_{j} g_{K} f_{i} \mu\right)$ $=E\left(\bar{f}_{j} f_{j} \mu\right)=E(\mu)$. Thus $h=\bar{f}_{j} g_{K} f_{i} \in \Lambda$ and $g_{K} f_{i}=f_{j} h$. We now have that

$$
\begin{aligned}
g_{K} h_{i} \mu & =g_{K}\left(\int_{\Lambda} f_{i} f d m\right) \mu=\left(\int_{\Lambda} g_{K} f_{i} f d m\right) \mu \\
& =\left(\int_{\Lambda} f_{j} h f d m\right) \mu=\left(\int_{\Lambda} f_{j} f d m\right) \mu=h_{j} \mu .
\end{aligned}
$$

Also, if $g_{K} h_{i} \mu=g_{K} h_{j} \mu$, then $g_{K} f_{i} \sim g_{K} f_{j}$, which implies that $f_{i} \sim f_{j}$. Thus the mapping $h_{i} \mu \rightarrow g_{K} h_{i} \mu$ is a permutation of the $\left\{h_{i} \mu\right\}$. Since supp $\sigma \subset \operatorname{supp} \mu$ and $|\mu|\left(T_{g_{K}}^{c}\right)=0$, we have that

$$
g_{K} \sigma=g_{K}\left(h_{1} \mu * \cdots * h_{n} \mu\right)=g_{K} h_{1} \mu * \cdots * g_{K} h_{n} \mu=\sigma .
$$

Now let $R=\left\{x \in \operatorname{supp} \mu:\left|\bar{\Psi}_{\beta}(x)-g_{K}(x)\right|<\sqrt{\delta}\right\}$. It follows from (5) that $|\mu|\left(R^{c}\right) \leqslant \sqrt{\delta}$. A result of Rider [10, Lemma 5.1] implies that, on $R^{n}=R$ $\times \cdots \times R$, we have the inequality

$$
\left|\Psi_{\beta}\left(x_{1} \cdots x_{n}\right)-g_{K}\left(x_{1} \cdots x_{n}\right)\right| \leqslant n^{2} \sqrt{\delta}, \quad x_{i} \in R(1<i<n) .
$$

Now

$$
\begin{aligned}
\left\|\left(\bar{\Psi}_{\beta}-1\right) \sigma\right\| & =\left\|\left(\bar{\Psi}_{\beta}-g_{K}\right) \sigma\right\| \\
& \leqslant \int\left|\bar{\Psi}_{\beta}\left(x_{1} \cdots x_{n}\right)-g_{K}\left(x_{1} \cdots x_{n}\right)\right| d(|\mu| \times \cdots \times|\mu|) \\
& \leqslant n^{2} \delta^{1 / 2} M^{n}+2 n M^{n-1} \delta^{1 / 2}<1 / 600
\end{aligned}
$$

for $\delta$ small enough. The second inequality above follows by integrating over $R^{n}$ and $\left(R^{n}\right)^{c}$ separately.

The following lemma extends a result of Glicksberg's [2] to compact groups. It is a generalization of Helson's translation lemma.

LEMMA 11. Let $G$ be a compact group and suppose $\Psi_{\alpha} \mu \rightarrow \omega, w^{*}$, where $\left\{\Psi_{\alpha}\right\} \subset \Gamma$ is an irreducible sequence. If $0 \neq \omega=\omega * \eta$, where $\eta$ is $H$-canonical, then $\left\{\Psi_{\alpha}\right\}$ is contained in a finite number of hypercosets of $\mathrm{H}^{\perp}$.

Proof. The proof follows Glicksberg exactly. Since $\omega=\omega * \eta$, it follows that $h \rightarrow \delta_{h} * \omega$ is norm-continuous on $H$. Let $\mu=\rho+\sigma$ be the Lebesgue decomposition of $\mu$ with respect to $|\omega|$ where $\rho \ll|\omega|$ and $\sigma \perp|\omega|$. Thus $h \rightarrow \delta_{h} * \rho$ is also norm-continuous on $H$, and therefore we can approximate $\rho$ in norm by $\int_{H} \delta_{h} * \rho$. $f(h) d m_{H}=\rho * f m_{H}$, where $f \in L_{1}^{z}(H)$. If the conclusion of the lemma is false, then $\Psi_{\alpha} f m_{H} \rightarrow 0, w^{*}$, by the Riemann-Lebesgue lemma, so that $\Psi_{\alpha}\left(\rho * f m_{H}\right) \rightarrow 0$, $w^{*}$, since $\{\alpha\}$ is an irreducible sequence. Thus $\Psi_{\alpha} \rho \rightarrow 0, w^{*}$, and $\omega=\lim \Psi_{\alpha} \sigma$. Now let $g$ be a $w^{*}$-cluster point of $\left\{\Psi_{\alpha}\right\}$ in $L^{\infty}(|\sigma|)$. Then $\omega=g \sigma$ but $\omega \perp \sigma$, a contradiction.

The following lemma can be found in Rider [10, p. 471].

LEMMA 12 (RIDER). Let $H$ be a closed normal subgroup of a compact group $G$. Suppose $\left\{\Psi_{\alpha}\right\}$ is a sequence in $\Gamma$ such that $\left|\Psi_{\alpha}(x)\right| \rightarrow 1$ a.e. $\left(m_{H}\right)$. Then, for a large enough, $\left|\Psi_{\alpha}(x)\right| \equiv 1$ on $H$. 
TheOREM 13. Let $G=A \times \Pi_{1}^{\infty} G_{i}$ and let $\mu \in I(G)$ be of b.r.t. in each coordinate. There exists an integer $N$ such that $\mu=\mu * m_{H}$, where $H=\Pi_{N+1}^{\infty} G_{i}$.

Thus $\mu$ can be considered as a measure on $A \times \Pi_{1}^{N} G_{i}$ and we can apply the results of $\$ 3$. Note that the theorem implies that $\mu$ is of b.r.t.

Proof. As noted at the beginning of this section, we can assume $\hat{\mu}(\alpha) \geqslant 1$ for all $\alpha \in E(\mu)$. If $\|\mu\|=1$, then $\mu$ is an idempotent and hence $\mu=\gamma m_{K}$, where $K=A_{0} \times \Pi_{1}^{\infty} G_{i}$ with $A_{0}$ a compact subgroup of $A$ and $\gamma \in \Gamma\left(A_{0}\right)$ (see Greenleaf [4, Theorem 2.1.4]). Now assume the theorem is true for all measures of norm less than $A$ and assume $1 \leqslant A \leqslant\|\mu\|<A+1 / 100 A$.

Assume the theorem is false. Using the fact that $\mu$ is of b.r.t. in each coordinate and that each $G_{i}$ satisfies Condition II, we obtain a sequence $\left\{\alpha_{n}\right\} \subset E(\mu)$ with the following properties.

(a) $\alpha_{n}=\beta_{1} \cdots \beta_{n} \gamma_{n} \lambda_{n}$, where $\beta_{i} \in \Gamma\left(G_{i}\right), \gamma_{n} \in \Gamma(A)$ and $\lambda_{n} \in \Gamma\left(\Pi_{n+1}^{\infty} G_{i}\right)$.

(b) $d\left(\lambda_{n}\right)>1$ for each $n$.

Let $P=\left\{x \in G:\left|\Psi_{\beta_{i}}(x)\right| \rightarrow 1\right.$ as $\left.i \rightarrow \infty\right\}$. Then $P$ is a normal Borel subgroup of $G$ which contains $A \times \Pi_{1}^{m} G_{i}$ for all $m$. We shall show that $P=G$ and hence, by Lemma 12, that $\Psi_{\beta_{\mathrm{i}}} \equiv 1$ for $i$ large enough. Now

$$
\int\left|\Psi_{\alpha_{n}}\right| d\left|\mu-\pi_{P} \mu\right| \rightarrow 0 \text { as } n \rightarrow \infty
$$

by the Lebesgue Dominated Convergence Theorem. Thus we can choose an $N$ such that

$$
\int\left|\Psi_{\alpha_{N}}\right| d\left|\mu-\pi_{P} \mu\right|<\frac{1}{4} \text {. }
$$

Define $K=A \times \Pi_{1}^{N^{\circ}} G_{i}$, where $N_{0}>N$ is chosen so that $\alpha_{N} \in \Gamma(K)$, and put $\lambda=\bar{\Psi}_{\alpha_{N}} \mu * m_{K}$. Then $1 \in E(\lambda), \lambda \in I(G)$ and $\lambda$ is of b.r.t. in each coordinate. If $\alpha \in E(\lambda)$, then

$$
\hat{\lambda}(\alpha)=\int \bar{\Psi}_{\alpha} d \lambda=\int \bar{\Psi}_{\alpha} \bar{\Psi}_{\alpha_{N}} d \pi_{P} \mu+\int \bar{\Psi}_{\alpha} \bar{\Psi}_{\alpha_{N}} d\left(\mu-\pi_{P} \mu\right) .
$$

From (6) and (7) it follows that $\left|\left(\pi_{P} \lambda\right)^{\wedge}(\alpha)\right| \geqslant 3 / 4$ if $\alpha \in E(\lambda)$. In particular, $E(\lambda) \subseteq E\left(\pi_{P} \lambda\right)$.

Let $B=\left\{\omega: \omega=w^{*}\right.$-lim $\Psi_{t_{n}} \lambda$, where $\left\{t_{n}\right\}$ is an $F$-sequence in $\left.E(\lambda)\right\}$. Since $1 \in E(\lambda), \varnothing \neq B \subseteq I(G)$. Also, $\omega \in B$ implies that $1 \in E(\omega)$. Using diagonal sequences one can show that $B$ is closed in the $w^{*}$ topology. Since $0 \notin B$, it follows that $B$ contains an element $\omega_{0}$ of minimal norm.

Suppose $\left\|\omega_{0}\right\|<A$. The induction hypothesis implies that $\omega_{0}$ is a trigonometric polynomial on $G$. If $\omega_{0}=\lim \bar{\Psi}_{t_{n}} \lambda$, then Lemma 11 implies that $\Psi_{t_{n}} \equiv 1$ for $n$ large enough, and hence $\omega_{0}=\lambda$. Since $\pi_{P} \lambda \neq 0$, it follows that $P$ is open in $G$ and thus $P=G$.

Now suppose $A \leqslant\left\|\omega_{0}\right\|<A+1 / 100 A$. If $\omega_{0}=\lim \bar{\Psi}_{t_{n}} \lambda$, a result of Rider [10, Lemma 7.4] shows that $\left\|\bar{\Psi}_{t_{n}} \lambda-\bar{\Psi}_{t_{m}} \lambda\right\|<1 / 4$ for $n, m$ large enough. Letting $m \rightarrow \infty$, we have the inequality $\left\|\Psi_{t_{n}} \lambda-\omega_{0}\right\|<1 / 4$, and hence

$$
\left\|\bar{\Psi}_{t_{n}} \pi_{P} \lambda-\pi_{P} \omega_{0}\right\| \leqslant 1 / 4 \text { for } n \text { large enough. }
$$


From (8) and the fact that $\left|\left(\pi_{P} \lambda\right)^{\wedge}(\alpha)\right| \geqslant 3 / 4$ if $\alpha \in E(\lambda)$, it follows that $E\left(\omega_{0}\right) \subseteq$ $E\left(\pi_{P} \omega_{0}\right)$.

Now let $B_{0}=\left\{\sigma: \sigma=w^{*}-\lim \bar{\Psi}_{t_{n}} \omega_{0}\right.$, where $\left\{t_{n}\right\}$ is an $F$-sequence in $\left.E\left(\omega_{0}\right)\right\}$. It is not difficult to show that $B_{0} \subseteq B$, and since $\omega_{0}$ was chosen of minimal norm in $B$, it follows that $\|\sigma\|=\left\|\omega_{0}\right\|$ if $\sigma \in B_{0}$. By Rider [10, Lemma 7.5], if $\sigma=w^{*}$ $\lim \bar{\Psi}_{t_{n}} \omega_{0} \in B_{0}$, then $\bar{\Psi}_{t_{n}} \omega_{0}$ converges to $\sigma$ in norm. Thus $\sigma=f \omega_{0}$, where (for a subsequence) $f(x)=\lim \bar{\Psi}_{t_{n}}(x)$, a.e. $\left(\left|\omega_{0}\right|\right)$. The function $f$ is a Borel homomorphism on a normal Borel subgroup of the support of $\omega_{0}$. Identify $B_{0}$ with the set of such $f$ and partition $B_{0}$ by $f \sim g$ if $E\left(f \omega_{0}\right)=E\left(g \omega_{0}\right)$. The number of equivalence classes is finite, for otherwise we would have a sequence $\left\{f_{i}\right\} \subseteq B_{0}$ with $E\left(f_{i} \omega_{0}\right) \neq E\left(f_{j} \omega_{0}\right)$ if $i \neq j$. By using a diagonal process, we can find an $f \in B_{0}$ such that (for a subsequence) $f_{i} \omega_{0} \rightarrow f \omega_{0}$ in norm. But then $\left\|f_{i} \omega_{0}-f_{j} \omega_{0}\right\|<1 / 2$ for $i$ and $j$ large enough. Since $\left(f_{i} \omega_{0}\right)^{\wedge}(\alpha) \geqslant 1$ for all $i$ and for all $\alpha \in E\left(f_{i} \omega_{0}\right)$, it follows that $E\left(f_{i} \omega_{0}\right)=E\left(f_{j} \omega_{0}\right)$, a contradiction.

Now choose $\delta$ as in Lemma 10 and choose $N_{1}>N_{0}$ such that if $\alpha=\alpha_{1} \cdots \alpha_{n}$ $\in E\left(\omega_{0}\right)$ and $\alpha_{i} \equiv 1$ for $i \leqslant N_{1}$, then $\left\|\bar{\Psi}_{\alpha} \omega_{0}-f \omega_{0}\right\|<\delta$ for some $f \in B_{0}$. Let $K_{1}=A \times \Pi_{1}^{N_{1} G_{i}}$ and put $\lambda_{0}=\omega_{0} * m_{K_{1}}$. Then $\lambda_{0} \neq 0$ and $\alpha \in E\left(\lambda_{0}\right)$ if and only if $\left\|\bar{\Psi}_{\alpha} \lambda_{0}-f \lambda_{0}\right\|<\delta$ for some $f \in B_{0}$. We shall now identify $\lambda_{0}$ with its projection on the group $\Pi_{N_{1}+1}^{\infty} G_{i}$. One can now show that the hypotheses of Lemma 10 are satisfied (applied to $\lambda_{0}$ and the group $\Pi_{N_{1}+1}^{\infty} G_{i}$ ), and thus obtain functions $h_{i} \in$ $L^{\infty}\left(\left|\lambda_{0}\right|\right)$ such that if $\sigma_{0}=h_{1} \lambda_{0} * \cdots * h_{n} \lambda_{0}$ then $\alpha \in E\left(\sigma_{0}\right)$ if and only if $\left\|\left(\bar{\Psi}_{\alpha}-1\right) \sigma_{0}\right\|<1 / 600$. Lemma 9 implies that $\sigma_{0}$ is a multiple of Haar measure of $\prod_{N_{1}+1}^{\infty} G_{i}$. Now recall that $E\left(\omega_{0}\right) \subseteq E\left(\pi_{P} \omega_{0}\right)$. This implies that if $\bar{\Psi}_{\alpha} \omega_{0} \rightarrow f \omega_{0}$ in norm, then $E\left(f \lambda_{0}\right) \subseteq E\left(f \pi_{P} \lambda_{0}\right)$, and thus $E\left(h_{i} \lambda_{0}\right) \subseteq E\left(h_{i} \pi_{P} \lambda_{0}\right)$ for $1 \leqslant i<n$. Therefore, $E\left(\sigma_{0}\right) \subseteq E\left(\pi_{P} \sigma_{0}\right)$ and hence $\pi_{P} \sigma_{0} \neq 0$. Thus $P$ is open in $\Pi_{N_{1}+1}^{\infty} G_{i}$, hence in $G$.

Lemma 12 now yields the existence of an $M$ such that $\Psi_{\beta_{i}} \equiv 1$ for all $i>M$. Let $\theta=\beta_{1} \cdots \beta_{M}$, let $K=\Pi_{1}^{M} G_{i}$ and let $\omega=w^{*}-\lim \bar{\gamma}_{\lambda}\left(\bar{\Psi}_{\theta} \mu * m_{K}\right)$ (for a subsequence). We shall identify $\omega$ with its projection on the group $A \times \Pi_{M+1}^{\infty} G_{i}$. We can repeat the argument given above beginning with the paragraph following (7) (and omitting the following two paragraphs), replacing $\lambda$ by the measure $\omega$. What was needed there was the fact that $1 \in E(\lambda)$ to ensure that $B \neq \varnothing$. We thus obtain a measure $\omega_{0}=\lim \Psi_{t_{n}} \omega$, where $\left\{t_{n}\right\}$ is an $F$-sequence in $E(\omega)$, and functions $h_{1}, \ldots, h_{n}$ such that if $\lambda_{0}=\omega_{0} * m_{K_{0}}$ and $\sigma=h_{1} \lambda_{0} * \cdots * h_{n} \lambda_{0}$, then $\alpha \in E(\sigma)$ if and only if $\left\|\left(\Psi_{\alpha}-1\right) \sigma\right\|<1 / 600$. Here $K_{0}=\Pi_{1}^{M_{\alpha}} G_{i}$, where $M_{0}>M$, and as usual $\sigma$ is identified with its projection on $A \times \Pi_{M_{0}+1}^{\infty} G_{i}$.

We claim now that, for some $M_{1} \geqslant M_{0}, \sigma=\sigma * m_{H}$, where $H=\Pi_{M_{1}}^{\infty} G_{i}$. To see this, we repeat the arguments in the first part of the proof to show the existence of an integer $I$ and a sequence $\left\{\alpha_{n}\right\} \subset E(\sigma)$ with $\alpha_{n}=\beta_{1} \cdots \beta_{I} \gamma_{n} \lambda_{n}$, where $\left\{\gamma_{n} \lambda_{n}\right\}$ forms an $F$-sequence. We can also assume $d\left(\lambda_{n}\right)>1$ for all $n$. Let $\beta=\beta_{1} \cdots \beta_{I}$ and choose integers $n_{1}$ and $n_{2}$ such that $\gamma_{n_{1}} \lambda_{n_{1}} \gamma_{n_{2}} \lambda_{n_{2}}$ is irreducible. Now

$$
\left|\int\left(\bar{\Psi}_{\beta}^{2} \bar{\gamma}_{n_{1}} \bar{\Psi}_{\lambda_{n_{1}}} \bar{\gamma}_{n_{2}} \bar{\Psi}_{\lambda_{n_{2}}}-1\right) d \sigma\right|<\left\|\left(\bar{\Psi}_{\beta} \bar{\gamma}_{n_{1}} \bar{\Psi}_{\lambda_{n_{1}}}-1\right) \sigma\right\|+\left\|\left(\bar{\Psi}_{\beta} \bar{\gamma}_{n_{2}} \bar{\Psi}_{\lambda_{n_{2}}}-1\right) \sigma\right\|<\frac{2}{600} \text {. }
$$

Thus, since $1 \in E(\sigma)$ and $\sigma \in I(G)$, there exists $\theta_{1} \in \beta \otimes \beta$ such that $\theta_{1} \gamma_{n_{1}} \lambda_{n_{1}} \gamma_{n_{2}} \lambda_{n_{2}}$ $\in E(\sigma)$. Hence, $\left\|\left(\bar{\Psi}_{\theta_{1}} \bar{\gamma}_{n_{1}} \bar{\Psi}_{\lambda_{n_{1}}} \bar{\Psi}_{n_{2}} \bar{\Psi}_{\lambda_{n_{2}}}-1\right) \sigma\right\|<1 / 600$, and we can repeat the argu- 
ment to show that for some $\theta_{2} \in \theta_{1} \otimes \beta$ and some $\gamma_{n_{3}} \lambda_{n_{3}}, \theta_{2} \gamma_{n_{1}} \lambda_{n_{1}} \gamma_{n_{2}} \lambda_{n_{2}} \gamma_{n_{3}} \lambda_{n_{3}} \in$ $E(\sigma)$. Thus we obtain a sequence $\left\{t_{m}\right\} \subseteq E(\sigma)$ with $t_{m}=\theta_{m} \gamma_{n_{1}} \lambda_{n_{1}} \gamma_{n_{2}} \lambda_{n_{2}} \cdots \gamma_{n_{m}} \lambda_{n_{m}}$. By repeating again the arguments in the first part of the proof, we see that $\lambda_{n_{m}} \equiv 1$ for $m$ large enough, a contradiction and our claim is established.

Now let $g=w^{*}-\lim \bar{\Psi}_{t_{n}}$ in $L^{\infty}(|\omega|)$. Then, since $C(G) \subseteq L^{\infty}(|\omega|)$, we have that

$$
\omega_{0}=g \omega=w^{*}-\lim \bar{\gamma} \bar{\Psi}_{\lambda_{n}}\left(g \mu_{0}\right)
$$

where $\mu_{0}=\Psi_{\theta} \mu * m_{K}$. Since $\left\{\gamma_{n} \lambda_{n}\right\}$ is an irreducible sequence,

$$
\lambda_{0}=\omega_{0} * m_{K_{0}}=w^{*}-\lim \bar{\gamma}_{n} \bar{\Psi}_{\lambda_{n}}\left(g \mu_{0} * m_{K_{0}}\right) \text {. }
$$

Also, for each $i$

$$
h_{i} \lambda_{0}=w^{*}-\lim \bar{\gamma}_{n} \bar{\Psi}_{\lambda_{n}}\left(h_{i} g \mu_{0} * m_{K_{0}}\right)
$$

so that

$$
\sigma=\prod h_{i} \lambda_{0}=w^{*}-\lim \bar{\gamma}_{n} \bar{\Psi}_{\lambda_{n}}\left(\prod h_{i} g \mu_{0} * m_{K_{0}}\right)
$$

Hence, by Lemma $11,\left\{\lambda_{n}\right\}$ is contained in finitely many hypercosets of $H$ which contradicts (b), and the theorem is proved.

Corollary 14. If $G=\Pi_{1}^{\infty} G_{i}$ and $\mu \in I(G)$ is of b.r.t. in each coordinate, then $\mu$ is a trigonometric polynomial.

5. The general case. In this section we use Weil's characterization of compact, connected groups to prove Theorem 1. This amounts to extending our results to abritrary products and to factor groups.

Proof of Theorem 1. (a) Arbitrary products. Let $\mu \in I(G)$, where $G=A \times$ $\Pi_{I} G_{\alpha}$ and where, as usual, $A$ is abelian and each $G_{\alpha}$ is a compact, connected, simple Lie group. Suppose that there exists a countable set $J \subseteq I$ and a countably infinite sequence $\left\{\alpha_{n}\right\} \subseteq \Gamma\left(\Pi_{J} G_{\alpha}\right)$ such that $\left\{\alpha_{n}\right\} \subset E(\mu)$ and $\Psi_{\alpha_{n} \mid \Pi_{J} G_{\alpha}}$ are all distinct. If $\nu=\mu * m_{K}$, where $K=\Pi_{I-J} G_{\alpha}$, then $\nu \neq 0, \nu^{*} \in I\left(A \times \prod_{J} G_{\alpha}\right)$, and $\left\{\alpha_{n}\right\} \subseteq E\left(\nu^{*}\right)$. If $\nu$ is of b.r.t. in each coordinate, then Theorem 13 implies that $\Psi_{\alpha_{n} \mid \Pi, G_{\alpha}}$ are not all distinct, a contradiction. Thus we can assume that $\mu$ is not of b.r.t. for all but a finite number of coordinates. Let $H=A \times \Pi_{I} H_{\alpha}$ where $H_{\alpha}=Z_{\alpha}$ if $\mu$ is not of b.r.t. in the $\alpha$ th coordinate and $H_{\alpha}=G_{\alpha}$ otherwise. We claim that $\sigma=\mu_{\mid H} \neq 0$ and that $\sigma \in I(H)$. This will complete the proof since we can then apply Theorem 6 to $\sigma$ and use the fact that $\pi_{K} \sigma=\pi_{K} \mu$ for $K$ a normal subgroup of $H$.

If $|\mu|(H)=0$ then the regularity of $\mu$ implies the existence of open sets $V_{n} \supset H$ such that $|\mu|\left(V_{n}\right)<1 / n$. Hence, $|\mu|\left(\cap_{1}^{\infty} V_{n}\right)=0$, and since each $V_{n}$ restricts only a finite number of coordinates, it follows that $|\mu|\left(A \times \Pi_{J} Z_{i} \times \Pi_{I-J} G_{\alpha}\right)=0$ where $J$ is countable. However, the results in the first two paragraphs of $\$ 4$ show that this is impossible since we are assuming that $\mu$ is of b.r,t. for only finitely many coordinates. 
To show that $\sigma \in I(H)$ let $\gamma \in \Gamma(H)$, let $\varepsilon>0$, and let $V$ be an open set containing $H$ such that $|\mu|(V-H)<\varepsilon$. If $\beta \in \Gamma(G)$ is chosen such that $\Psi_{\beta \mid H}=$ $\gamma$, then

$$
\int \bar{\gamma} d \sigma=\int \bar{\Psi}_{\beta} d \mu-\left.\int \bar{\Psi}_{\beta} d \mu\right|_{G-V}-\left.\int \Psi_{\beta} d \mu\right|_{V-H} .
$$

The last integral on the right has absolute value less than $\varepsilon$. Since $V$ restricts only a finite number of coordinates, $\beta$ can be chosen (with $d(\beta)$ large) so as to make the second integral less than $\varepsilon$ (see the third paragraph in \$4). Since $\mu \in I(G)$, it follows that $\sigma \in I(H)$.

(b) Factor groups. If $G$ is a group for which Theorem 1 is valid and $H$ is a closed, normal subgroup of $G$, then Theorem 1 is valid for $G / H$. Let $\sigma \in I(G / H)$ and choose $\mu \in I(G)$ such that $\mu^{*}=\sigma$ and $\mu * m_{H}=\mu$. There exists a closed, normal subgroup $K$ of $G$ such that $0 \neq \pi_{K} \mu=\nu * \eta$ where $\nu$ is invertible and $\eta$ is $K$-canonical. Now

$$
0 \neq \pi_{K} \mu=\pi_{K}\left(\mu * m_{H}\right)=\pi_{K} \mu * m_{H} .
$$

This implies that $K$ is open in $H K$ and thus $\pi_{K} \mu=\pi_{H K} \mu$. By Lemma 3 we have the equality

$$
\pi_{H K / H} \sigma=\left(\pi_{K} \mu\right)^{*}=(\nu * \eta)^{*}=\nu^{*} * \eta^{*} .
$$

By comparing transforms it is easy to see that $\nu^{*}$ is invertible in $M(G / H)$ and that $\eta^{*} \ll m_{H K / H}$.

6. A counterexample. Theorem 1 fails for the disconnected case. Let $G$ be the semidirect product $T \times T \times \mid Z_{2}$, where $T$ is the circle and $Z_{2}$ the group of order 2 . $Z_{2}$ acts on $T \times T$ by $\alpha\left(t_{1}, t_{2}\right)=\left(t_{2}, t_{1}\right)$ for $\alpha \neq e$. Let $\mu_{1}$ be Haar measure on $T \times e \times e, \mu_{2}$ Haar measure on $e \times T \times e$ and $\mu_{3}$ Haar measure on $T \times T \times e$. If $\mu=\mu_{1}+\mu_{2}-\mu_{3}$ then $\mu$ is a central idempotent. Suppose there exists a normal subgroup $H$ of $G$ for which $\pi_{H} \mu=\nu * \eta \neq 0$. It is easily seen that the only normal subgroup $G$ for which $\pi_{H} \mu \neq 0$ is $T \times T \times e$ which has finite index in $G$. Thus $\mu=\pi_{H} \mu=\nu * \eta=\eta$ since $\eta$ and $\mu$ are both idempotents. However, this implies that $\mu \ll m_{T \times T \times e}$ which is not true.

\section{REFERENCES}

1. I. Amemiya and T. Ito, A simple proof of the theorem of P. J. Cohen, Bull. Amer. Math. Soc. 70 (1964), 774-776.

2. I. Glicksberg, Fourier-Stieltjes transforms with an isolated value, Conference on Harmonic Analysis (Univ. Maryland, College Park, Md., 1971), Lecture Notes in Math., Vol. 266, Springer, Berlin, 1972, pp. 59-72.

3. I. Glicksberg and I. Wik, The range of Fourier-Stielties transforms of parts of measures, Conference on Harmonic Analysis (Univ. Maryland, College Park, Md., 1971), Lecture Notes in Math., Vol. 266, Springer, Berlin, 1972, pp. 73-77.

4. F. P. Greenleaf, Norm decreasing homomorphism of group algebras, Pacific J. Math. 15 (1965), 1187-1219.

5. E. Hewitt and K. A. Ross, Abstract harmonic analysis. Vol. I, Springer-Verlag, Berlin and New York, 1963.

6. Abstract harmonic analysis. Vol. II, Springer-Verlag, Berlin and New York, 1970. 
7. C. C. Moore, Groups with finite dimensional irreducible representations, Trans. Amer. Math. Soc. 166 (1972), 401-410.

8. D. L. Ragozin, Central measures on compact simple Lie groups, J. Functional Analysis 10 (1972), 212-229.

9. D. Rider, Central idempotent measures on unitary groups, Canad. J. Math. 22 (1970), 719-725.

10. Central idempotent measures on compact groups, Trans. Amer. Math. Soc. 186 (1973), 459-479.

11. W. Rudin, Fourier analysis on groups, Interscience, New York, 1962.

12. A. Weil, L'intégration dans les groupes topologiques et ses applications, Actualités Sci. Indust., Hermann, Paris, 1938, pp. 869-1145.

Department of Mathematics, Lafayette College, Easton, Pennsylvania 18042 